\title{
1 Immobilized Aptamer Paper Spray Ionization Source for Ion Mobility 2 Spectrometry
}

3

4

\author{
Tahereh Zargar, Taghi Khayamian,* and Mohammad T. Jafari \\ Department of Chemistry, Isfahan University of Technology, Isfahan 84156-83111, Iran \\ Corresponding Author *Phone: +98-311-391-3273. Fax: +98-311-391-2350. E-mail: taghi@cc.iut.ac.ir.
}

A selective thin-film microextraction based on aptamer immobilized on cellulose paper was used as a paper spray ionization source for ion mobility spectrometry (PSI-IMS), for the first time. In this method, the paper is not only used as an ionization source but also it is utilized for the selective extraction of analyte, based on immobilized aptamer. This combination integrates both sample preparation and analyte ionization in a Whatman paper. To that end, an appropriate sample introduction system with a novel design was constructed for the paper spray ionization source. Using this system, a continuous solvent flow works as an elution and spray solvent simultaneously. In this method, analyte is adsorbed on a triangular paper with immobilized aptamer and then it is desorbed and ionized by elution solvent and applied high voltage on paper, respectively. The effects of different experimental parameters such as applied voltage, angle of paper tip, distance between paper tip and counter electrode, elution solvent type, and solvent flow rate were optimized. The proposed method was exhaustively validated in terms of sensitivity and reproducibility by analyzing the standard solutions of codeine and acetamiprid. The analytical results obtained are promising enough to ensure the use of immobilized aptamer 
1 paper-spray as both the extraction and ionization techniques in IMS for direct analysis of

2 biomedicine.

3 Keywords: Paper spray ionization, Aptamer, ion mobility spectrometry

\section{Introduction}

5 Paper spray ionization (PSI), is an ambient ionization source, introduced by Cooks and Ouyang

6 in 2010 [1]. This ionization technique, was used for analysis of samples in their native

7 environment in the open air to the mass spectrometer (MS), with no or little requirement

8 for sample preparation or preseparation [2]. In the PSI, sample is loaded onto a triangular

9 piece of paper and ions are generated by applying a high voltage to the wetted paper for the

10 analysis. The influences of various parameters such as geometry of the paper, onset voltage for

11 spray, on the ionization efficiency of paper spray have been investigated by Cooks [3]. He

12 reported a paper spray method for analysis of polar analytes using non-polar solvents.

13 Continuous solvent have been applied to improve the stability of signal [4]. On the other hand,

14 the capability of paper spray in analyzing target analytes with different sample matrices has been

15 systematically characterized by Ouyang [5]. Paper is readily available at very low cost in

16 different types and has a great potential to be used as an inexpensive diagnostics [6].

17 Chromatographic grade paper showed the best analytical performance in chemical analysis [7].

18 Paper spray using chromatography paper is a new ionization method which integrates three 19 analytical procedures: sample storage, analyte separation, and analyte ionization [8].

While the main attractions of paper spray ionization are its low cost and easy to use,

21 its combination with ion mobility spectrometry (IMS) is more convenient than MS, where the

22 former is much cheaper than a MS. Although the small portable IMS analyzers are limited in 
1 resolving power, selectivity of a chemical determination can be reliable if the ionization

2 chemistry for the analyte overcomes matrix interferences [9]. In 2011, Sukumar et al. [10]

3 showed the capability of paper spray ionization for a drift tube ion mobility spectrometer. A

4 prototype ion mobility spectrometer coupled with a paper spray ionization source was also

5 developed by Jie et al [9]. In 2014, this group reported a new PSI-IMS method for direct, rapid,

6 and in-situ detection of analyte residues both in liquid samples and on solid surfaces [11]. Also

7 in 2015, Manicke et al. used field asymmetric ion mobility spectrometry with paper spray

8 ionization source for analysis of opiate isomers [12].Paper spray analysis with an integrated solid

9 phase extraction (SPE) column for sample pre concentration, extraction and ionization was also

10 reported [13].

Recently, coupling of SPME with MS including SPME with wooden tips and paper

12 spray has been reviewed [14]. Pawliszyn research group also used the coated blade spray (CBS)

13 as a solid-substrate for the extraction and ionization source in MS [15].

Thin film microextraction (TFME) as a mode of SPME was introduced by Pawliszyn

15 research group in 2003 [16]. In TFME, a thin and wide membrane is employed as a sorbent for

16 microextraction procedures. The TFME has great extraction efficiency due to the large volume

17 of its extraction phase and its high surface-to-volume ratio. Chemical modification of the

18 cellulose surface with the suitable organic reagents makes it appropriate for extraction purposes.

19 In 2013, Saraji et al developed surface modification of cellulose paper with different chemical

20 reagents [17]. Aptamer-based TFME method as a sorbent for the extraction of codeine has been

21 reported in our group [18]. In that work, the amino-modified aptamer was immobilized on the

22 oxidized cellulose surface through a covalent bond. 
2 of codeine and acetamiprid as the test compounds from different categories (drug and pesticide,

3 respectively) and then the same papers were used as the ionization source in IMS.

4 Therefore, the selective extraction integrates with the ionization source, providing a specific

5 and highly sensitive extraction method for the fast and online analysis of analytes in real

6 complex matrices. The influence of different experimental parameters such as applied voltage,

7 angle of paper tip, distance between paper tip-counter electrode, elution solvent type and solvent

8 flow rate was investigated.

\section{Experimental section}

\section{2.1. Chemicals}

12 Codeine, oxycodone, and morphine were prepared from Darou Pakhsh (Tehran, Iran).

13 Papaverine was purchased from Alborz Daroo (Tehran, Iran). Imidacloprid and Chlorpyrifos

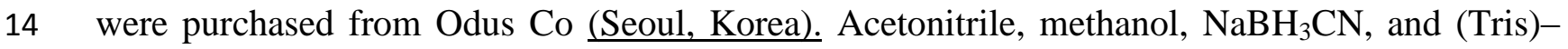
$15 \mathrm{HCl}$ were purchased from Merck (Darmstadt, Germany). Analytical grade of $\mathrm{MgCl}_{2}, \mathrm{KCl}$, $16 \mathrm{Na}_{2} \mathrm{HPO}_{4} \cdot 2 \mathrm{H}_{2} \mathrm{O}, \mathrm{KH}_{2} \mathrm{PO}_{4}$, and $\mathrm{NaIO}_{4}$ were obtained from Riedel-de Haën (Seelze, Germany). 17 The 5'-amino-modified DNA $\quad$ oligonucleotides $\quad$ (sequence5'18 CCCCCTGGGTCGGGAGGGAAGGGGGTTG GGGGTGCGG-3' as the anticodeine aptamer 19 with a $\mathrm{C}_{6}$ spacer arm) were synthesized by Metabion (Planegg, Germany). The sequence of the 20 anticodeine aptamer used in this work has been reported by Shangguan et al. [19] Aptamer with 21 the sequence of 5'-TGT AAT TTG TCT GCA GCG GTT CTT GAT CGC TGA CAC CAT ATT 22 ATG AAG A-3'was chosen according to the previously reported literature (Heet al., 2011) [20]. 
1 The 5'-amino-modified aptamer stock solution was prepared in $25 \mathrm{mM}$ Tris- $\mathrm{HCl}$ buffer solution $2 \quad(\mathrm{pH} 7.4)$ and stored at $4^{\circ} \mathrm{C}$.

5 Cellulose ashless filter paper of grade 44 was purchased from Whatman (Maidstone, UK). For

6 paper spray, the paper substrate was cut into a triangle (14 $\mathrm{mm}$ height and $4 \mathrm{~mm}$ base width).

7 Paper as a thin film was prepared according to previously reported procedures [18]. Briefly

8 Triangular-shaped piece of paper was placed in a vacuum desiccator. Then the paper was

9 oxidized with $\mathrm{NaIO}_{4}$ and aldehyde groups are formed on the surface. In order to stabilize the

10 aptamer on aldehyde paper, the amino-modified aptamer was transferred to a glass vial

11 containing four pieces of aldehyde-modified cellulose paper. Then, $\mathrm{NaBH}_{3} \mathrm{CN}$ was added to it.

12 The reaction continued by shaking the solution overnight at room temperature in the dark. In the

13 end, any non-covalently bound aptamer was removed by washing the pieces of aldehyde

14 modified cellulose paper with Tris- $\mathrm{HCl}$ solution at $\mathrm{pH} 7.4$ and deionized water for three times.

$16 \quad$ 2.3. TFME procedure

17 The extraction procedure has been described in reference [18]. In summary, at first anticodeine

18 or antiacetamiprid immobilized aptamer cellulose paper was attached to a stainless steel cutter

19 and together were named "sampler". After that, the sampler was immersed into a solution of

20 Tris- $\mathrm{HCl}$ buffer for 5 minutes for conditioning the paper and it was dried at room temperature.

21 Then, the sampler was immersed into a glass vial with $15 \mathrm{~mL}$ of analyzing sample. In order to 
1 keep the paper fix inside the glass vial, a polyethylene disk with a diameter equal to the inner

2 diameter of the screw cap of the vial was used. The paper was incubated for 30 minutes at room

3 temperature under shaking (400 rpm; KS 260 basic, IKA, Staufen, Germany). Then the sampler

4 was withdrawn from the glass vial and inserted into the glass tube and the paper was separated to

5 be used in the paper spray.

\subsection{Ion Mobility Spectrometry}

8 The electrospray ionization ion mobility spectrometer (ESI-IMS) used for this research was 9 constructed at Isfahan University of Technology [21]. In brief, the IMS cell was divided to two 10 sections: a desolvation region and a drift region, separated by a shutter grid. A pulse generator at 11 a frequency of $25 \mathrm{~Hz}$ removed the grid potential for a short period of time to give an ion pulse to

12 the drift region. $\mathrm{N}_{2}$ gas was used as the drift and the desolvation gases with flow rates of 500 and

$13900 \mathrm{~mL} \mathrm{~min}{ }^{-1}$, respectively. A high-speed analog-to digital converter module (12-bit dynamic

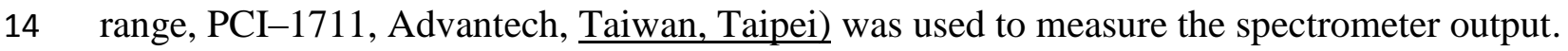

\section{$16 \quad 3$. Results and discussion}

\section{3.1. Designed sample introduction system}

18 A new sample introduction system was designed and constructed for the PSI-IMS. The 19 schematic diagram of the apparatus including sample introduction system is shown in Fig. 1. It 20 consists of a paper holder, solvent inlet, and a screw for high voltage connection. The holder was

21 fabricated by machining two brass pieces with a $200 \mu \mathrm{m}$ gap between them. Two metal pieces of 
1 the paper holder joined together by a screw. Each metal piece has a $10 \mathrm{~mm}$ diameter and $5 \mathrm{~mm}$

2 thickness. In the upper part of the paper holder, a hole was made to insert a Teflon tube for

3 delivering solvent. In this system, solvent is continuously delivered on the paper as an elution

4 and spray solvent. The paper is inserted into the paper holder, where about $2 \mathrm{~mm}$ of the paper tip

5 protrudes from the metal pieces. The metal pieces were connected to a PTFE ring to change its

6 position along the IMS cell axis, for adjusting its distance relative to the counter electrode.

\subsection{Ion Mobility Spectrum}

9 Fig. 2 (a) shows the ion mobility spectrum of the background obtained using the immobilized aptamer paper spray without the analytes. This spectrum shows only ion peaks originated from

11 paper spray solvent with a considerable intensity. The ion mobility spectrum of eluted codeine 12 and acetamiprid, where they were desorbed from the aptamer are also shown in Fig. 2 (b and c).

13 Based on this figure, codeine and acetamiprid produces two $\left(C_{1}\right.$ and $\left.C_{2}\right)$ and three $\left(A_{1}, A_{2}\right.$, and $14 A_{3}$ ) ion peaks, respectively, during the paper spray process. Using the standard compound of 15 nicotinamide $\left(\mathrm{K}_{0}=1.85 \mathrm{~cm}^{2} \mathrm{~V} \cdot \mathrm{s}^{-1}\right)$, the reduced mobility values were obtained to be 1.14 and 1.10 $16 \mathrm{~cm}^{2} \mathrm{~V} \cdot \mathrm{s}^{-1}$ for $\mathrm{C}_{1}$ and $\mathrm{C}_{2}$, and $1.40,1.11$, and $0.95 \mathrm{~cm}^{2} \mathrm{~V} \cdot \mathrm{s}^{-1}$ for $\mathrm{A}_{1}, \mathrm{~A}_{2}$, and $\mathrm{A}_{3}$, respectively.

\subsection{PSI parameters}

19 The Paper was inserted between the metal pieces of the paper holder and the high voltage was connected to the screw of the lower part of the paper holder. The distance between the paper tip

21 and counter was changed from 3 to $9 \mathrm{~mm}$ and $7 \mathrm{~mm}$ was selected as the optimized distance (Fig. 
13 ). Acetonitrile-water (90:10, v/v) and acetonitrile were the optimized solvents for the elution of

2 codeine and acetamiprid, respectively. The solvent was delivered continuously to the paper at the

3 flow rate of $24 \mu \mathrm{L} \mathrm{min}{ }^{-1}$. When the solution was reached to the paper tip, the paper spray

4 phenomenon was occurred and its corresponding signal was observed in ion mobility spectrum.

5 The IMS analysis was carried out in the positive ion mode with a spray voltage at $14.1 \mathrm{kV}$.

\subsection{Extraction conditions}

8 Generally, extraction is improved with increasing the extraction time. The effect of the 9 extraction time on the extraction efficiencies was investigated in the range of 10-50 min. The 10 time required to reach equilibrium were 30, $40 \mathrm{~min}$ for codeine and acetamiprid, respectively. As 11 we know, the rise of temperature leads to increase mobility of the species in the sample solution 12 and accelerates the mass transfer process. Temperatures between 10 to $50{ }^{\circ} \mathrm{C}$ with intervals of 10 13 degrees were studied and $30{ }^{\circ} \mathrm{C}$ was selected as the optimized temperature (Fig. 3).

14 Another parameter with a critical effect on both extraction and ionization efficiency is the 15 composition of the solvent. In this proposed method, the solvent fed on the immobilized aptamer 16 paper has two very important roles. From one side, the solvent should be able to elute the analyte 17 with an acceptable efficiency. On the other hand, the selected solvent should have the appropriate properties to establish a stable paper spray plume. In this work, the best response for

19 elution of codeine was obtained by a mixture of acetonitrile-water with a ratio of 90:10 (v/v) and 20 the elution solvent for acetamiprid was pure acetonitrile for the subsequent experiments (Fig. 3). 
2 The analytical capability of the proposed method was studied by analysis of codeine and

3 acetamiprid. The area of the ion peak was considered as the response of the PSI-IMS. The linear

4 range was found to be $10-500 \mathrm{ng} \mathrm{mL}^{-1}$ of codeine with the detection limit of $3.7 \mathrm{ng} \mathrm{mL}^{-1}$ and the

5 dynamic range was obtained from 5 to $300 \mathrm{ng} \mathrm{mL}^{-1}$ for acetamiprid with the detection limit of

$6 \quad 1.8 \mathrm{ng} \mathrm{mL} \mathrm{mL}^{-1}$. The relative standard deviation was calculated for triplicate measurement of

7 different concentrations of both analytes and its range was obtained from $2.1 \%$ to $5.6 \%$.

8 In order to show the selectivity of the aptameres, the homologues of the analytes with

9 approximately the same reduced mobilities were selected. The spectra and reduced mobilities of

10 the homologues and interferences are shown and listed in Fig.4. and Table1, respectively. The

11 results of their intensities with the same concentration of the analyte are also shown in Fig.5.

12 These results demonstrated the selectivity of the aptamers for capturing the analytes from the

13 samples. In addition, the results showed that solvent delivery can desorb the analytes from the

14 aptamers and they ionized by paper spray.

15 3.6. Analysis of real samples

16 The application of the method was shown by analysis of codeine in spiked urine samples and

17 also codeine tablets from different pharmaceutical companies. The results of recoveries for

18 codeine spiked samples and analysis of codeine in codeine tablets showed the capability of the

19 method for analysis of codeine in these samples. The method was also used for analysis of

20 antiacetamiprid in vegetable samples.

21 3.6.1 Analysis of codeine in pharmaceutical samples 
1 In order to confirm the capability of the method for analyzing of real samples, tablets from five

2 companies with concentrations of acetaminophen-codeine 300/10 $\mathrm{mg}$ were selected and the

3 codeine concentrations were determined by the proposed method. At first, 10 tablets for each

4 company were grounded and mixed individually and then the concentration was measured in the

5 powder. The results are shown in Table $2 \mathrm{a}$. The statistical results of t-test show that there is no

6 significant difference between the reported and the measured amounts of codeine.

7

$8 \quad$ 3.6.2. Analysis of codeine in urine sample

9 Codeine at three concentration levels in urine samples was investigated by this method to

10 evaluate the performance of the method in analysis of real samples. To investigate the effect of

11 the urine matrix on the analytical signal, the relative recovery was calculated. The relative

12 recoveries were found to be $87.3,90.7$ and $88.6 \%$ for the urine samples spiked with codeine at

13 three concentrations of 50,100 and $200 \mathrm{ng} / \mathrm{mL}$. The RSD values were under $6.4 \%$ for three

14 replicate measurements.

16 3.6.3. Analysis of acetamiprid in different samples

17 Detection of acetamiprid in wastewater, tomato and cucumbers from environmental and 18 agricultural samples were also carried out for demonstration of method ability. The extraction 19 from the wastewater was performed by filtering through a $0.22 \mu \mathrm{m}$ filter. Cucumbers and 20 tomatoes were first homogenized to a free-flowing puree in a mortar and then they were 21 centrifuged for $20 \mathrm{~min}$ at $1000 \mathrm{rpm}$ to remove the solids, and finally the supernatants were 
1 filtered through a $0.22 \mathrm{~mm}$ filter. The recoveries for the extractions were evaluated by the

2 standard addition method using three different concentrations. Table $2 \mathrm{~b}$ shows the recovery 3 results of acetamiprid in the three matrices.

\section{Conclusion}

6 A novel method based on integration of sample preparation and ionization source was introduced

7 using a Whatman paper. The paper was used as an ionization source and as a TFME, with a

8 selective biorecognition aptamer. A new sample introduction system was also designed and

9 constructed for continuously deliver solvent on the paper spray as the elution and paper spray

10 solvent. The performance of the proposed method was evaluated by analyzing codeine and

11 acetamiprid in pharmaceutical, environmental and agricultural samples. This method provides a

12 selective, fast, and simple technique for direct analysis of analytes in real samples. Furthermore,

13 it opens a new horizon for implication of paper spray as an integration of ionization source and 14 selective extraction for clinical studies, point of care methods, and food safety environmental 15 monitoring.

\section{Acknowledgment}

18 The authors are grateful for financial support of this work by the Research Council of Isfahan 19 University of Technology (IUT), Iran, and the Center of Excellency in Chemistry of Isfahan 20 University of Technology. The authors thank the Iran National Science Foundation (grant no. 21 93030712) for its support. 


\section{References}

[1] H. Wang, J. Liu, R.G. Cooks, Z. Ouyang, Paper spray for direct analysis of complex mixtures using mass spectrometry, Angew. Chem. Int. Ed. 122 (2010) 889-892.

[2] R.D. Espy, A.R. Muliadi, Z. Ouyang, R.G. Cooks, Spray mechanism in paper spray ionization, Int. J. Mass Spectrom. 325 (2012) 167-171.

[3] Q. Yang, H. Wang, J.D. Maas, W.J. Chappell, N.E. Manicke, R.G. Cooks, Z. Ouyang, Paper spray ionization devices for direct, biomedical analysis using mass spectrometry, Int. J. Mass Spectrom. 312 (2011) 201-207.

[4] A. Li, H. Wang, Z. Ouyang, R.G. Cooks, Paper spray ionization of polar analytes using non-polar solvents, chem. commun. 47 (2011) 2811-2813.

[5] J. Liu, H. Wang, N.E. Manicke, J.M. Lin, R.G. Cooks, Z. Ouyang, Development, characterization, and application of paper spray ionization, Anal. Chem. 82 (2010) 2463-2471.

[6] A.W. Martinez, S.T. Phillips, G.M. Whitesides, Three-dimensional microfluidic devices fabricated in layered paper and tape, Proc. Natl. Acad. Sci.U. S. A. 105 (2008) 19606-19611.

[7] J. Sherma, B. Fried, Thin-layer and paper chromatography, Anal. Chem. 54 (1982) 45R-57R.

[8] N.E. Manicke, Q. Yang, H. Wang, S. Oradu, Z. Ouyang, R.G. Cooks, Assessment of paper spray ionization for quantitation of pharmaceuticals in blood spots, Int. J. Mass Spectrom. 300 (2011) 123129.

[9] Z. Jingjing, J. Jie, Q. Xiaolin, Development of an ion mobility spectrometer with a paper spray ionization source, Rev. Sci. Instrum. 84 (2013) 065103.

[10] H. Sukumar, J.A. Stone, T. Nishiyama, C. Yuan, G.A. Eiceman, Paper spray ionization with ion mobility spectrometry at ambient pressure, Int. J. Ion Mobil Spectrom 14 (2011) 51-59.

[11] M. Li, J. Zhang, J. Jiang, J. Gao, X. Qiao, Rapid, in situ detection of cocaine residues based on paper spray ionization coupled with ion mobility spectrometry, Analyst 139 (2014) 1687-1691.

[12] N.E. Manicke, M. Belford, Separation of opiate isomers using electrospray ionization and paper spray coupled to high-field asymmetric waveform ion mobility spectrometry, J. Am. Soc. Mass. Spectrom. 26 (2015) 701-705.

[13] C. Zhang, N.E. Manicke, Development of a paper spray mass spectrometry cartridge with integrated solid phase extraction for bioanalysis, Anal. Chem. 87 (2015) 6212-6219.

[14] J. Deng, Y. Yang, X. Wang, T. Luan, Strategies for coupling solid-phase microextraction with mass spectrometry, TrAC, Trends Anal. Chem. 55 (2014) 55-67.

[15] G.A. Gómez-Ríos, J. Pawliszyn, Development of coated blade spray ionization mass spectrometry for the quantitation of target analytes present in complex matrices, Angew. Chem. Int. Ed. 53 (2014) $\underline{14503-14507 .}$

[16] I. Bruheim, X. Liu, J. Pawliszyn, Thin-film microextraction, Anal. Chem. 75 (2003) 1002-1010. 
1

3

4

5

6

7

8

9

5

26

8

9

[17] M. Saraji, B. Farajmand, Chemically modified cellulose paper as a thin film microextraction phase, J. Chromatogr. A 1314 (2013) 24-30.

[18] Z. Hashemian, T. Khayamian, M. Saraji, Anticodeine aptamer immobilized on a Whatman cellulose paper for thin-film microextraction of codeine from urine followed by electrospray ionization ion mobility spectrometry, Anal. Bioanal. Chem. 407 (2015) 1615-1623.

[19] L. Huang, X. Yang, C. Qi, X. Niu, C. Zhao, X. Zhao, D. Shangguan, Y. Yang, A label-free electrochemical biosensor based on a DNA aptamer against codeine, Anal. Chim. Acta 787 (2013) 203-210.

[20] J. He, Y. Liu, M. Fan, X. Liu, Isolation and identification of the DNA aptamer target to acetamiprid, Agric. Food. Chem. 59 (2011) 1582-1586.

[21]T. Khayamian, M.T. Jafari, Design for electrospray ionization-ion mobility spectrometry, Anal. Chem. 79 (2007) 3199-3205.

0

1

2

3

4

7




\section{$1 \quad$ Figure captions}

2 Fig. 1. The schematic diagram of the paper holder and paper spray ionization source in the PS-

3 IMS apparatus.

4 Fig. 2. The ion mobility spectra of solvent (a), codeine $\left(100 \mathrm{ng} \mathrm{mL}^{-1}\right)(\mathrm{b})$ and acetamiprid (100

$5 \mathrm{ng} \mathrm{mL} \mathrm{L}^{-1}$ ) using the PSI-IMS.

6 Fig. 3. (a) The response of different tip angles on paper spray ionization; (b) The effect of 7 distance between the paper tip and counter electrode; (c) The effect of time on the extraction

8 efficiency of the analytes; (d) The effect of temperature on the extraction efficiency of the 9 analytes; (e) The effect of spray solvent on the analysis of the analytes at $100 \mathrm{ng} \mathrm{mL}{ }^{-1}$ 10 (concentration of analytes: $100 \mathrm{ng} \mathrm{mL}^{-1}$; extraction temperature: $30^{\circ} \mathrm{C}$ ).

11 Fig. 4. Ion mobility spectra for the analytes and their interferences at $100 \mathrm{ng} \mathrm{mL}^{-1}$.

12 Fig. 5. The response of the PS-IMS for codeine (above) and acetamiprid (below) and their 13 homologues and interferences at the same concentrations. 
Table 1. The reduced mobility values $\left(\mathrm{K}_{0}\right)$ for the analytes and their homologues and interferences.

\begin{tabular}{llll}
\hline $\begin{array}{l}\text { Compound } \\
\text { (Drugs) }\end{array}$ & $\mathrm{K}_{0}\left(\mathrm{~cm}^{2} \mathrm{~V} . \mathrm{s}^{-1}\right)$ & $\begin{array}{l}\text { Compound } \\
\text { (Pesticides) }\end{array}$ & $\begin{array}{l}\mathrm{K}_{0}\left(\mathrm{~cm}^{2}\right. \\
\left.\mathrm{V} . \mathrm{s}^{-1}\right)\end{array}$ \\
\hline Codeine & 1.14 & Acetamiprid & 1.40 \\
& 1.10 & & 1.11 \\
& & & 0.95 \\
Morphine & 1.13 & Imidacloprid & 1.49 \\
& & & 1.13 \\
Papaverine & 1.09 & Chlorpyrifos & 1.31 \\
Oxycodone & 1.14 & & \\
\hline
\end{tabular}


Table 2. (a)The analysis of codeine in pharmaceutical samples using the proposed method; (b) analysis of acetamiprid in different samples using PSI-IMS.

(a)

\begin{tabular}{lllll}
\hline Company & $\begin{array}{l}\text { Claim } \\
(\mathrm{mg})\end{array}$ & $\begin{array}{l}\text { Found } \\
(\mathrm{mg})\end{array}$ & Recovery\% & $\begin{array}{l}\text { RSD\% } \\
(\mathrm{n}=3)\end{array}$ \\
\hline Khwarizmi & 10 & 9.43 & 94.3 & 2.1 \\
Hakim & 10 & 9.76 & 97.6 & 1.9 \\
Exir & 10 & 11.07 & 110.7 & 2.4 \\
Luqman & 10 & 8.53 & 85.3 & 3.5 \\
Tehranchemie & 10 & 8.76 & 87.6 & 2.2 \\
\hline
\end{tabular}

(b)

\begin{tabular}{lllll}
\hline Sample & $\begin{array}{l}\text { Amount added } \\
\left.(\mathrm{ng} \mathrm{mL})^{-1}\right)\end{array}$ & $\begin{array}{l}\text { Amount found } \\
\left(\mathrm{ng} \mathrm{mL}^{-1}\right)\end{array}$ & Recovery\% & $\begin{array}{l}\text { RSD\% } \\
(\mathrm{n}=3)\end{array}$ \\
\hline & 0 & Undetected & & 4.3 \\
Wastewater & 50 & 44.7 & 89.4 & 2.9 \\
& 100 & 88.9 & 88.9 & \\
& 0 & Undetected & & 3.6 \\
Tomato & 50 & 46.3 & 92.6 & 6.4
\end{tabular}

$\begin{array}{cllll} & 0 & \text { Undetected } & \\ \text { Cucumber } & 50 & 50.6 & 101.2 & 5.1 \\ & 100 & 86.5 & 86.5 & 2.3\end{array}$




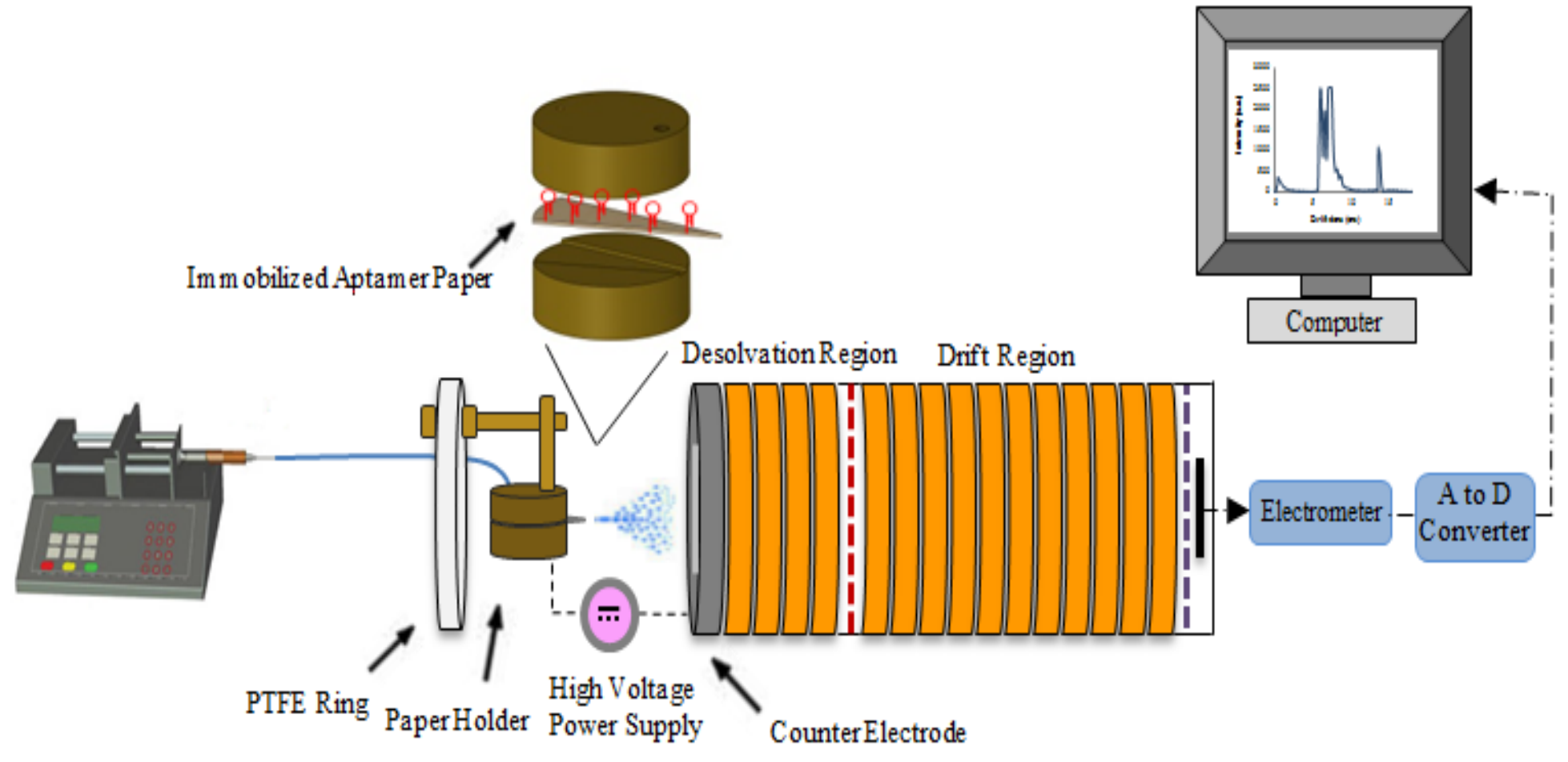

Fig. 1 
Figure(s)

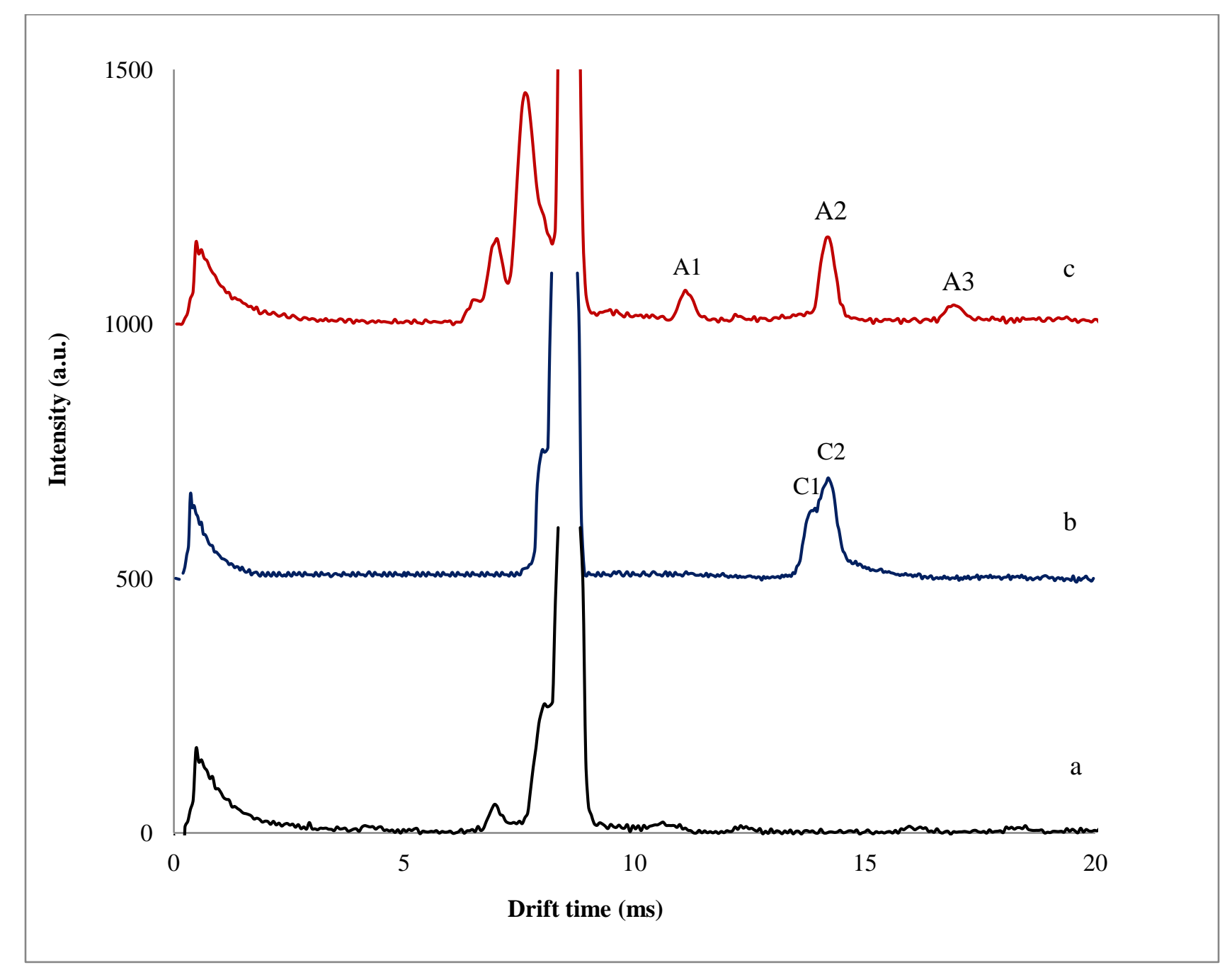

Fig. 2 

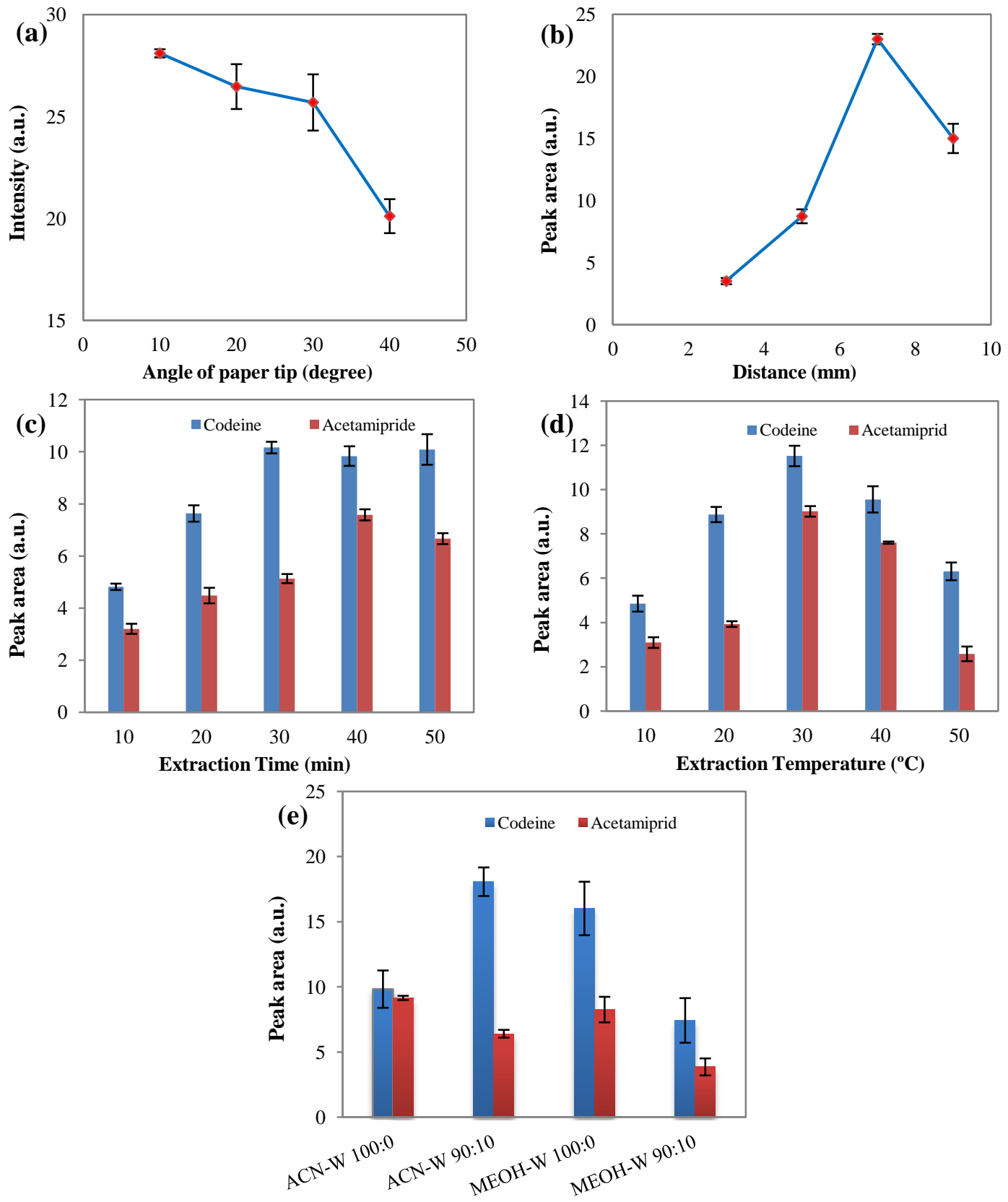

Fig. 3 

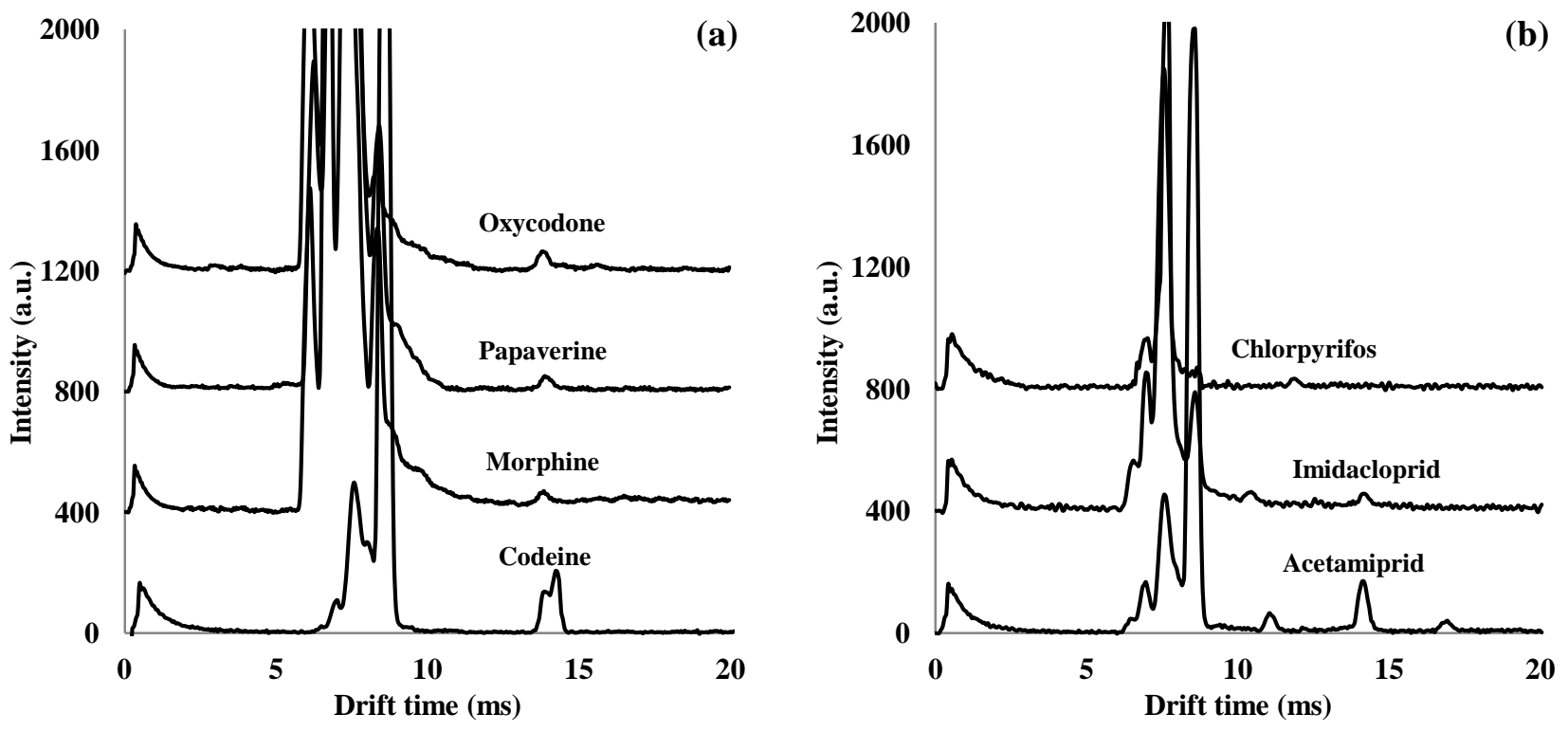

Fig. 4 

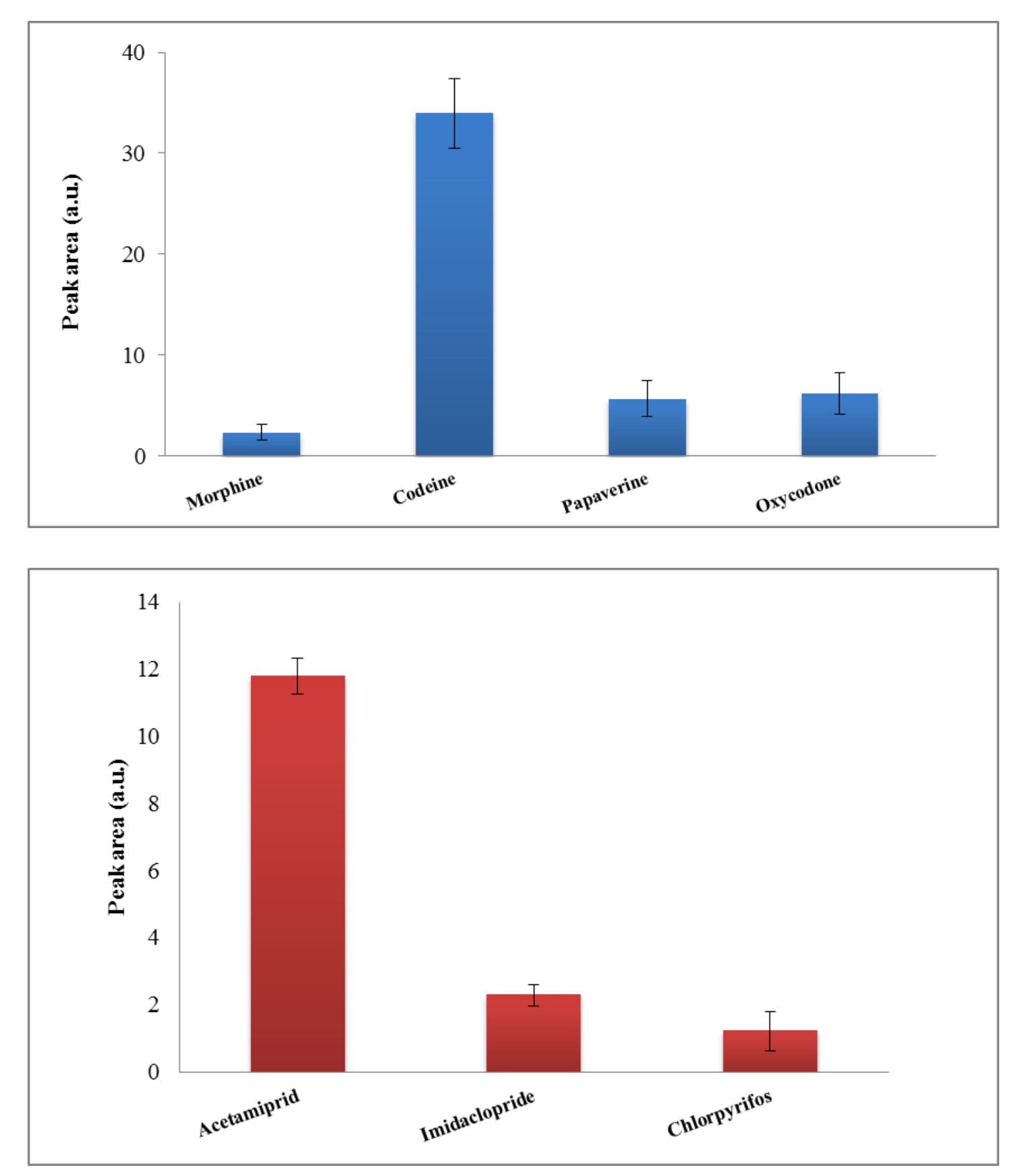

Fig. 5

Figure(s)
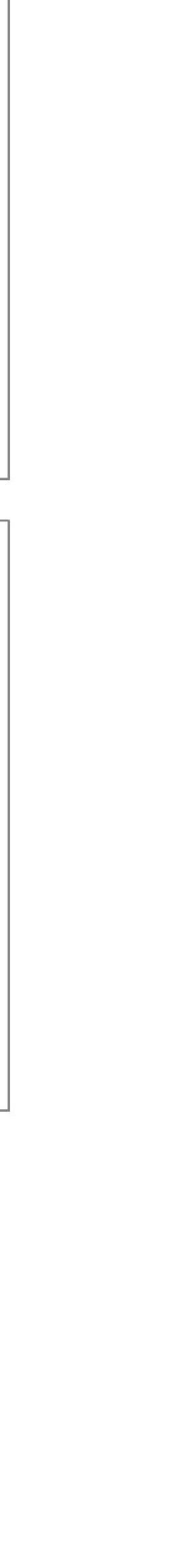

Figure

5
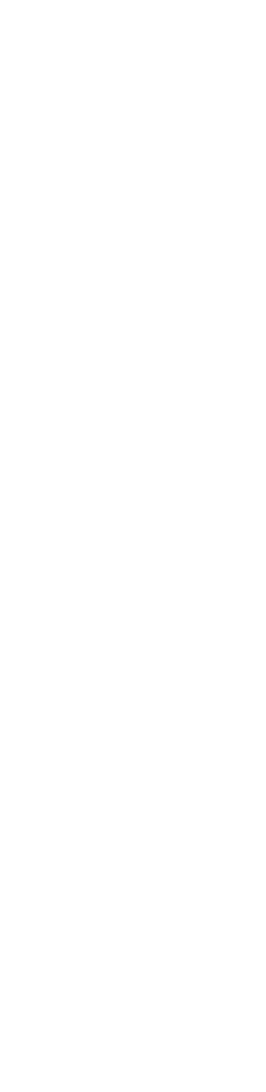
Graphical Abstract

\section{Graphical Abstract}

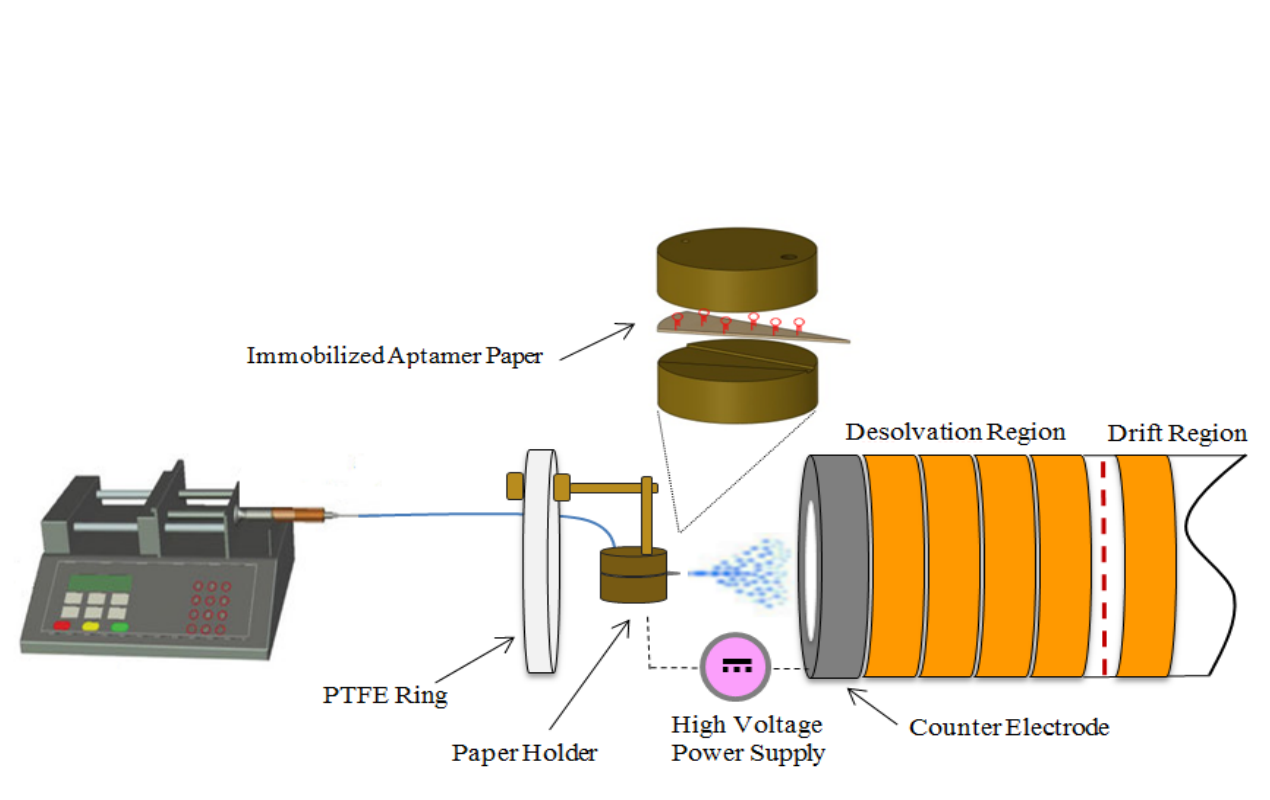

Graphical Abstract 